\title{
Erratum: Caroli formalism in near-field heat transfer between parallel graphene sheets [Phys. Rev. B 96, 155437 (2017)]
}

\author{
Jia-Huei Jiang and Jian-Sheng Wang
}

(Received 1 February 2021; published 19 March 2021)

DOI: 10.1103/PhysRevB.103.119902

This erratum aims to correct (1) the wrong expressions, (2) some typographical errors, (3) some erroneous points made in a discussion of the disparity of heat flux ratios between our full random phase approximation (RPA) model and the local conductivity model, and (4) the lower bound of the characteristic distance scale comparable to the graphene thermal length or Fermi wavelength, appearing in the original paper, which might confuse or mislead readers. Collectively, they do not change the key physics we sought to present.

First, we would like to correct some wrong expressions:

(1) In Eq. (A1), the electronic annihilation and creation operators were given incorrectly in the paper. They should be as follows: $c_{\mathbf{k}}^{(l)}=\left(c_{A \mathbf{k}}^{(l)}, c_{B \mathbf{k}}^{(l)}\right)^{T} ; c_{\mathbf{k}}^{\dagger(l)}=\left(c_{A \mathbf{k}}^{\dagger(l)}, c_{B \mathbf{k}}^{\dagger(l)}\right)$. This does not affect our calculations, presentation of key physics, or conclusions for this was only an accidental misimplementation during the drafting phase of the paper.

(2) The statement $\Pi^{r} \rightarrow \sum_{j, j^{\prime}} \Pi_{j j^{\prime}}^{r}$ above Eq. (D1) should be restated as $\Pi^{r} \rightarrow \frac{1}{4} \sum_{j, j^{\prime}} \Pi_{j j^{\prime}}^{r}$. The division by 4 is to give the sense of averaging over four components of $\Pi_{j j^{\prime}}^{r}$, when the $A$ and $B$ sublattices are indistinguishable. This statement is not used elsewhere in the paper and, therefore, does not affect our calculations, presentation of key physics, or conclusions.

Second, the typographical errors are corrected as below:

(1) The expressions for the $f(\mathbf{k})$ function appearing in Eqs. (A1) and (7) have the wrong signs for $k_{x}$ in the second and third terms. They should be as follows:

$$
f(\mathbf{k})=e^{-i k_{x} a_{0}}+e^{i k_{x} a_{0} / 2+i \sqrt{3} k_{y} a_{0} / 2}+e^{i k_{x} a_{0} / 2-i \sqrt{3} k_{y} a_{0} / 2} .
$$

(2) The expression for $q_{z}$ should have $\lim _{\tilde{c} \rightarrow \infty}$, instead of $\lim _{\tilde{c} \rightarrow 0}$.

(3) In Eq. (B3), the denominators on the right-hand side should be multiplied by $a_{0}$ to make the dimensions match for the Laplacian.

(4) In Eq. (D3), the $z^{\prime}$ and $z$ are taken to be $d^{-}$in the end $\left(z^{\prime}=z=d^{-}\right)$.

Third, we reclarify some points in discussing the disparity of heat flux ratios between our full RPA model and the local conductivity model:

(1) We inappropriately adopted the analytical expressions for the plasmon branches ( $\omega_{L}$ and $\omega_{H}$ [1]) valid only for highly doped sheets deviated by a mild temperature difference [see Eq. (E1) below with $\mu \gg k_{B} T$ ]. The inappropriateness can be obviously seen in the parameters of doping as light as $0.1 \mathrm{eV}$ and temperatures as 300 and $1000 \mathrm{~K}$ used in our original paper. To remedy this, we derive a more general form,

$$
\omega_{L / H}=\left[Z_{\text {avg }}\left(1 \mp \sqrt{1-\left(\frac{Z_{1}}{Z_{\text {avg }}}\right)\left(\frac{Z_{2}}{Z_{\text {avg }}}\right)\left(1-e^{-2 q d}\right)}\right)\right]^{1 / 2}, \quad q>0,
$$

where the $\omega_{L}$ corresponds to the minus branch and $\omega_{H}$ the positive; $Z_{\text {avg }}=\left(Z_{1}+Z_{2}\right) / 2 ; Z_{l}=\left(e^{2} k_{B} T_{l} / \epsilon_{0} \pi \hbar^{2}\right)$ $\ln \left[2 \cosh \left(\mu_{l} / 2 k_{B} T_{l}\right)\right] q$ [2]; the $\eta_{1}(=0.0033 \mathrm{eV})$ was neglected because it is very small compared to the energy spans we have chosen. One can readily verify that this formula goes back to the form $\omega_{L / H}=\omega_{s} \sqrt{1 \mp e^{-q d}}, \omega_{s}=\sqrt{Z_{\text {avg }}}$, when $Z_{1}=Z_{2}=Z_{\text {avg }}$ [1]. One can also show that $\omega_{L} \rightarrow \sqrt{\left(Z_{1} Z_{2} / Z_{\text {avg }}\right) q d}$ as $q d \rightarrow 0$. This way of estimating plasmon branches considers the Drude part of conductivity only, which is fair when the frequency (or $q$ ) is small and is dominant in a wider frequency span when $\mu$ is larger than $k_{B} T$ [2].

The purpose for drawing the $\omega_{L}$ and $\omega_{H}$ lines using Eq. (E1) above is for approximating the orientations of the two branches on the $\omega-q$ plane. The approximation can tell the initial orientations of the plasmon branches in the low-frequency regime within the local conductivity model.

We replace the cyan and green lines in Figs. 8 and 9 of our original paper with Eq. (E1) above and give the updated plots as follows (the figure indexing follows the same figure indexing in the original paper).

(2) The values of "critical distances" $d_{c}$ 's are nearly the same as previously reported in our original paper so we do not change them in light of Eq. (E1). 


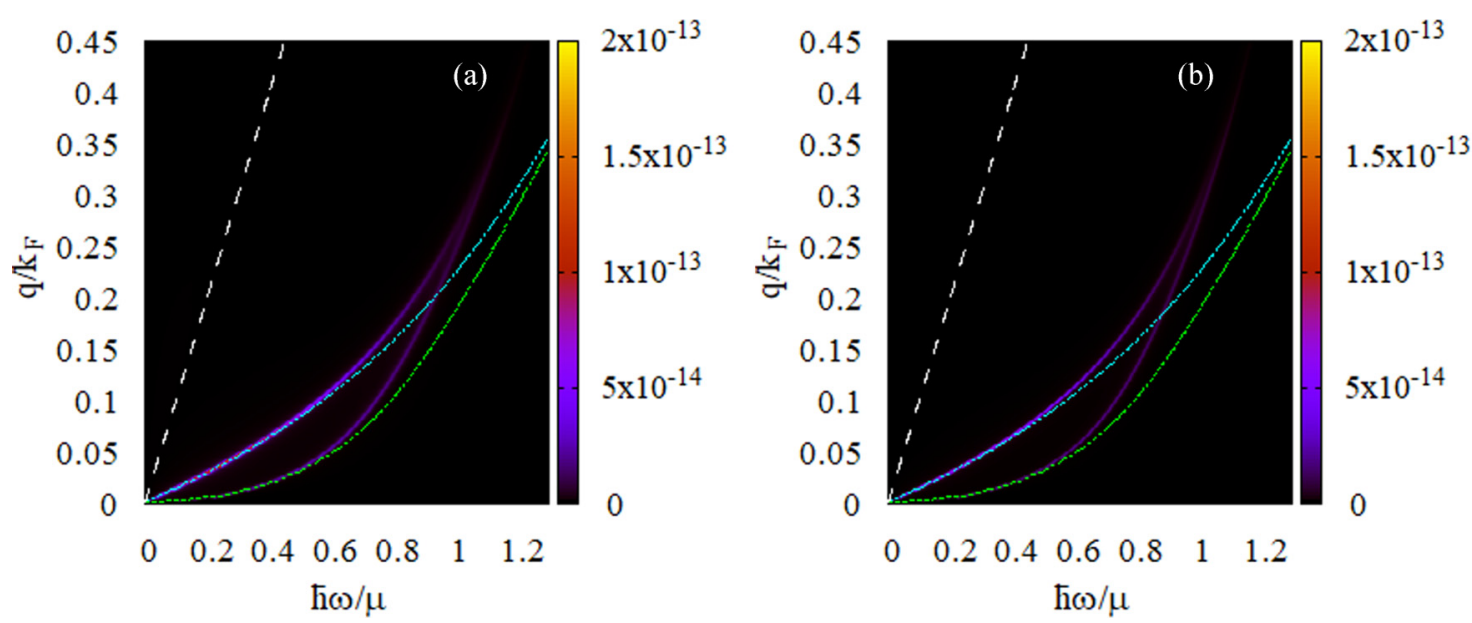

FIG. 8. Updated (a) and (b) with the cyan and green dashed lines based on Eq. (E1) replotted; curves standing for Eq. (E1) in the $\mu \gg k_{B} T$ limit are not plotted because the results closely overlap with the cyan and green dashed lines, respectively; the white dashed line marks the $\omega=v_{F} q$ border; other contents are the same as the original paper.
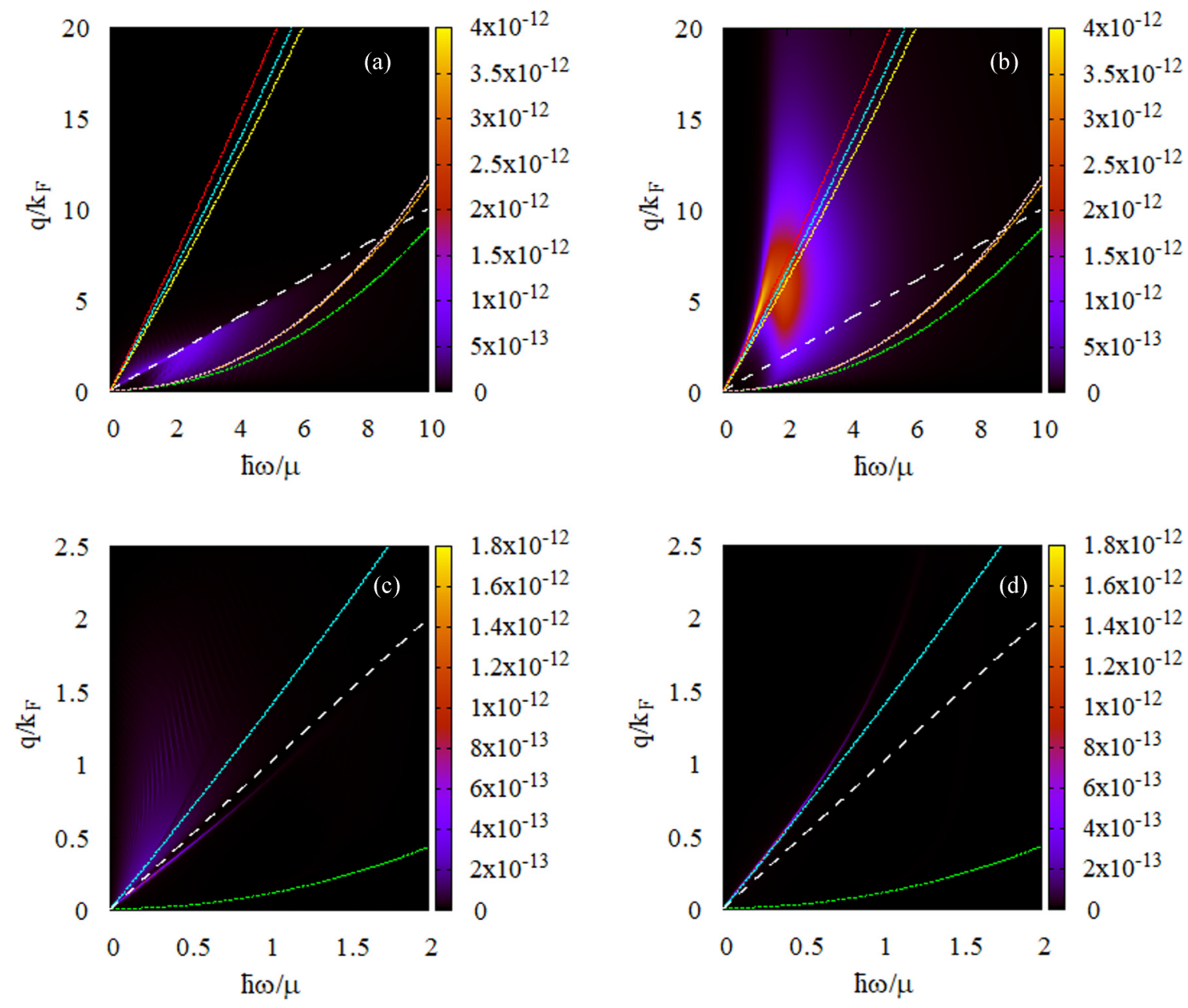

FIG. 9. Updated (a)-(d) with the cyan and green dashed lines based on Eq. (E1) replotted; the red and orange dashed lines stand respectively for $\omega_{L}$ and $\omega_{H}$ of Eq. (E1) in the $\mu \gg k_{B} T$ limit [these lines are not plotted for (c) and (d) because the results closely overlap with the cyan and green dashed lines, respectively]; the yellow and pink dashed lines were mistakenly and respectively taken as the red and orange dashed lines in the original paper; the white dashed line marks the $\omega=v_{F} q$ border; other contents are the same as the original paper. 
(3) We report the misplacement of the cyan $\omega_{L}$ and green $\omega_{H}$ lines drawn in Figs. 9(a) and 9(b) in the original paper. The correct lines can be obtained from Eq. (E1) and with the $\mu \gg k_{B} T$ limit applied [see the red, orange, yellow, and pink dashed lines Figs. 9(a) and 9(b) in this erratum]. Also, we report the misplacement of figures: Figs. 8(a) and 8(b) in the original paper were mistakenly interchanged due to the ultrasimilarity (the local-conductivity curves should bend more slightly inwards toward the $\omega=v_{F} q$ border and appear less blurry at the high-frequency ends).

(4) Our previous statement in the original paper, "The full RPA calculation of ours has rescued the extinction of acoustic plasmon mode under local conductivity approximation by constraining the mode to stay within the border of the $\omega=v_{F} q$ line," is not well phrased and confusing, for the full RPA calculation does not need to rescue its acoustic plasmon mode from invalidating the basic assumption $\left(\omega>v_{F} q\right)$ that the local conductivity model took. The better statement is as follows: "The full RPA calculation of ours seems to make the acoustic mode line stay within the border of the $\omega=v_{F} q$ line."

Fourth, the lower bound of the characteristic distance scale comparable to the graphene thermal length or Fermi wavelength should be set to $1 \mathrm{~nm}$ (it was $10 \mathrm{~nm}$ in the original paper), corresponding to $\hbar v_{F} / 0.7 \mathrm{eV} \simeq 0.942 \mathrm{~nm}(0.7 \mathrm{eV}$ is our highest energy parameter). We made this wrong estimation because we did not notice the importance of dividing the estimated wavelengths by $2 \pi$ when it no longer indicates a phase of a full cycle of wave in the exponent of $e^{-\left|q_{z}\right| d}$. We keep the upper bound (100 nm) unchanged, for the characteristic distance scale is now changed to $\hbar v_{F} /\left(k_{B} \times 300 \mathrm{~K}\right) \simeq 25.5 \mathrm{~nm}\left(k_{B} \times 300 \mathrm{~K}(\simeq 26 \mathrm{meV})\right.$ is our lowest energy parameter). Thus the range of the characteristic distance scale is now $1-100 \mathrm{~nm}$. It is checked that the correction only re-adjust our original statement and does influence other parts of the work.

[1] H. Iizuka and S. Fan, Analytical treatment of near-field electromagnetic heat transfer at the nanoscale, Phys. Rev. B 92, 144307 (2015).

[2] O. Ilic, M. Jablan, J. D. Joannopoulos, I. Celanovic, H. Buljan, and M. Soljačić, Near-field thermal radiation transfer controlled by plasmons in graphene, Phys. Rev. B 85, 155422 (2012). 